\title{
Luteolin and sorafenib combination kills human hepatocellular carcinoma cells through apoptosis potentiation and JNK activation
}

\author{
XU-QIN FENG ${ }^{1}$, LI-WEN RONG ${ }^{1}$, RUI-XUE WANG ${ }^{2}$, XUE-LIAN ZHENG ${ }^{2}$, LEI ZHANG $^{2}$, \\ $\mathrm{LIN}_{\text {ZHANG }}^{2}$, YONG $\mathrm{LIN}^{2}$, XIA WANG ${ }^{2}$ and ZHI-PING LI ${ }^{1}$
}

\author{
${ }^{1}$ Department of Abdomen Oncology, Cancer Center of West China Hospital, West China Medical School; \\ ${ }^{2}$ Laboratory of Molecular and Translational Medicine, Key Laboratory of Birth Defects and Related Diseases of \\ Women and Children (Sichuan University) of Ministry of Education, Department of Obstetrics and Gynecology, \\ West China Second University Hospital, Sichuan University, Chengdu, Sichuan 610041, P.R. China
}

Received April 26, 2016; Accepted August 23, 2017

DOI: $10.3892 / \mathrm{ol} .2018 .8640$

\begin{abstract}
Sorafenib is a small-molecule multi-kinase inhibitor approved by FDA as an oral agent for the treatment of hepatocellular carcinoma (HCC) and renal cell carcinoma. However, unresponsiveness and acquired resistance are commonly observed, which hinder the clinical use of sorafenib. As combination therapy is a promising approach to improve its efficacy, we investigated if sorafenib and luteolin combination is effective in killing human HCC cells. Cell death was examined by lactate dehydrogenase (LDH) releasing assay. Apoptosis was detected by flow cytometric. The activation of apoptotic pathway and c-Jun N-terminal kinase (JNK) signaling pathway was measured by western blot. The results showed that sorafenib and luteolin combination synergistically induced cytotoxicity in HCC cells, which was accompanied by potentiation of apoptosis as demonstrated by increased apoptotic cell populations, caspase activation, and suppression of cell death by the pan-caspase inhibitor z-VAD-fmk. Furthermore, the combination of both agents enhanced expression of phosphorylated form of JNK, and the JNK inhibitor SP600125 effectively attenuated cell death induced by the combination
\end{abstract}

Correspondence to: Professor Zhi-Ping Li, Department of Abdomen Oncology, Cancer Center of West China Hospital, West China Medical School, Sichuan University, 37 Guo Xue Xiang Road, Chengdu, Sichuan 610041, P.R. China

E-mail: lizhiping620312@163.com

Professor Xia Wang, Laboratory of Molecular and Translational Medicine, Key Laboratory of Birth Defects and Related Diseases of Women and Children (Sichuan University) of Ministry of Education, Department of Obstetrics and Gynecology, West China Second University Hospital, Sichuan University, 20 Renminnanlu San Duan Road, Chengdu, Sichuan 610041, P.R. China

E-mail: xiawang@scu.edu.cn

Key words: luteolin, sorafenib, apoptosis, c-Jun N-terminal kinase, hepatocellular carcinoma, drug resistance, synergy treatment. Thus, sorafenib and luteolin combination synergistically kills HCC cells through JNK-mediated apoptosis, and luteolin may be an ideal candidate for increasing the activity of sorafenib in HCC therapy.

\section{Introduction}

Sorafenib is a small-molecule multi-kinase inhibitor approved by the US Food and Drug Administration as an oral agent for the treatment of hepatocellular carcinoma (HCC) and renal cell carcinoma. It is an inhibitor of Raf serine/threonine kinases, c-Raf, wild type and mutant B-Raf, which are all essential constituents of the Ras/Raf/mitogen-activated protein kinase (MAPK) signaling pathway that is important for cancer cell proliferation, and survival. Sorafenib also potently inhibits receptor tyrosine kinases, including vascular endothelial growth factor receptor (VEGFR)-2 and -3, and the platelet-derived growth factor receptor- $\beta$. As these targets are involved in cancer growth and angiogenesis, sorafenib has been demonstrated to exert anticancer activity through inhibiting tumor cell proliferation, and tumor angiogenesis $(1,2)$. Two placebo-controlled phase III studies have revealed that sorafenib treatment results in $\sim 3$-month extension of survival in patients with advanced HCC $(3,4)$. Another phase III study on advanced HCC demonstrated a significant improvement in progression-free survival relative to the placebo group (167 vs. 84 days) (5). However, similar to other kinase inhibitors, unresponsiveness and acquired resistance are commonly observed in patients with $\mathrm{HCC}$, which hinder the clinical use of sorafenib $(6,7)$. Although the precise mechanism underlying sorafenib resistance remains elusive, various efforts have been made to improve sorafenib sensitivity, among which combination therapy is a promising approach.

Luteolin, 3',4',5,7-tetrahydroxyflavone, is a flavonoid identified in a variety of vegetables, including broccoli, green peppers and celery. Flavonoids are common nutrients that are antioxidants, estrogenic regulators and antimicrobial agents. Numerous flavonoids may also serve as cancer preventive agents (8). Likewise, luteolin has been demonstrated to exhibit anticancer activities, including the induction of apoptosis, cell cycle arrest and antiangiogenesis (9-11). The pro-apoptotic 
property of luteolin is associated with redox regulation, DNA damage and protein kinases inhibition (12). Furthermore, numerous studies have revealed that luteolin may sensitize cancer cells to therapeutically induced cytotoxicity through suppressing cell survival pathways and stimulating apoptosis pathways (13-15). Luteolin are generally safe and associated with low toxicity, making them ideal candidates for chemosensitizer. To the best of our knowledge, the combination effect of luteolin and sorafenib in cancer cell killing remains elucidated. In the present report, whether the combination of sorafenib and luteolin was able to increase anticancer activity in HCC cells was investigated, and the underlying mechanism was addressed.

\section{Materials and methods}

Reagents. Sorafenib (Nexavar ${ }^{\circledR}$ ) and luteolin were purchased from Bayer AG (Leverkusen, Germany) and Sigma-Aldrich (Merck KGaA, Darmstadt, Germany), respectively. Antibodies against active caspase-3 and poly (ADP-ribose) polymerase (PARP) were from BD Pharmingen (BD Biosciences, Franklin Lakes, NJ, USA). Anti-phospho-c-Jun N-terminal kinase (JNK), -JNK1, and - $\beta$-actin were from Cell Signaling Technology, Inc. (Danvers, MA, USA), Abcam (Cambridge, UK), and Protein Tech Group, Inc. (Chicago, IL, USA), respectively. The pan-caspase inhibitor zVAD-fmk, the JNK inhibitor SP600125, the ERK inhibitor U0126, and the P38 inhibitor SB203580 were purchased from Calbiochem (Merck KGaA). Reactive oxygen species (ROS) scavenger butylated hydroxyanisole (BHA) and N-acetyl-L-cysteine (NAC) were from Sigma-Aldrich (Merck KGaA).

Cell lines and cell culture. Human hepatocellular carcinoma Hep3B and SMMC-7721 cell lines were purchased from the Type Culture Collection of Chinese Academy of Sciences (Shanghai, China). The cells were cultured in Dulbecco's modified Eagle's medium (Invitrogen; Thermo Fisher Scientific, Inc., Waltham, MA, USA) supplemented with $10 \%$ fetal bovine serum (Sigma-Aldrich; Merck KGaA), 100 U/ml penicillin, and $100 \mu \mathrm{g} / \mathrm{ml}$ streptomycin under standard incubation conditions $\left(37^{\circ} \mathrm{C}, 5 \% \mathrm{CO}_{2}\right)$.

Cytotoxicity assay based on the release of lactate dehydrogenase $(L D H)$. Following designated treatment, cell death was quantitatively detected by a cytotoxicity assay based on the release of lactate dehydrogenase using a cytotoxicity detection kit (Promega Corporation, Madison, WI, USA) as described previously (16). Briefly, $50 \mu \mathrm{l}$ culture medium from each well was collected and transferred to 96 -well flat-bottomed plates. LDH activity was determined by adding $50 \mu 1$ reaction mixture to each well. The absorbance of the samples was measured at $490 \mathrm{~nm}$ using a plate reader. All the experiments were repeated between three and five times, and data are expressed as the mean \pm standard deviation. Cell death was calculated using the following formula: Cytotoxicity $(\%)=($ Experimental value-Spontaneous LDH release)/(Maximum LDH releaseSpontaneous LDH release) x 100.

Apoptosis analysis by flow cytometry. Flow cytometry was applied to detect apoptosis in cultured cells using an
Annexin V-fluorescein isothiocyanate (FITC) Apoptosis Detection kit purchased from Nanjing KeyGen Biotech Co., Ltd. (Nanjing, China). Following designated treatments, cells were double stained with annexin V-FITC and propidium iodide (PI) according to the manufacturer's protocol. Apoptosis was then analyzed by flow cytometry (BD Biosciences). Early apoptosis was defined as Annexin $\mathrm{V}^{+} / \mathrm{PI}^{-}$staining (B4) and late apoptosis was defined as Annexin $\mathrm{V}^{+} / \mathrm{PI}^{+}$staining (B2).

Western blot analysis. Cells treated as indicated in each figure legend were lysed in $\mathrm{M} 2$ buffer (20 mmol/l Tris- $\mathrm{HCl}(\mathrm{pH} 7.6)$, $0.5 \% \mathrm{NP} 40,250 \mathrm{mmol} / 1 \mathrm{NaCl}, 3 \mathrm{mmol} / 1$ EDTA, $3 \mathrm{mmol} / 1$ EGTA, $2 \mathrm{mmol} / 1 \mathrm{DTT}, 0.5 \mathrm{mmol} / 1$ phenylmethylsulfonyl fluoride, $20 \mathrm{mmol} / 1 \beta$-glycerophosphate, $1 \mathrm{mmol} / 1$ sodium vanadate, and $1 \mu \mathrm{g} / \mathrm{ml}$ leupeptin). Protein concentrations in the cell extracts were determined by using BCA Protein Assay Kit. Cell extracts $(\sim 50 \mu \mathrm{g})$ were resolved in SDS-PAGE $(8 \%$ gel for detecting PARP, $10 \%$ gel for detecting p-JNK, JNK1 and $\beta$-actin, and $15 \%$ gel for detecting active caspase 3 ), and then transferred to polyvinylidene fluoride membrane. The membrane was blocked with TBS containing 5\% milk and $0.05 \%$ Tween- 20 for $2 \mathrm{~h}$ at $37^{\circ} \mathrm{C}$ and detected with various antibodies: Anti-PARP (dilution 1:500; catalog no. 556494; BD Pharmingen; BD Biosciences; overnight at $4^{\circ} \mathrm{C}$ ); anti-p-JNK (dilution 1:1,500; catalog no. 9255; Cell Signaling Technology, Inc., overnight at $4^{\circ} \mathrm{C}$ ); anti-JNK1 (dilution 1:1,000; catalog no. 110724; Abcam, overnight at $4^{\circ} \mathrm{C}$ ); anti- $\beta$-actin (dilution 1:5,000, catalog no. 60008-1-Ig; Protein Tech Group, Inc.; 1 h at room temperature); and anti-active-caspase 3 (dilution 1:500; catalog no. 559565; BD Pharmingen; BD Biosciences; overnight at $4^{\circ} \mathrm{C}$ ). Subsequently, the membrane was washed three times for 5 min each with TBS containing Tween-20. Next, peroxidase-conjugated goat anti-mouse immunoglobulin (Ig) G (catalog no. ZB 2305; Beijing Zhongshan Golden Bridge Biotechnology Co., Ltd., Beijing, China) or peroxidase-conjugated goat anti-rabbit IgG (catalog no. ZB 2301; Beijing Zhongshan Golden Bridge Biotechnology Co., Ltd.) was added at a 1:5,000 dilution and incubated with the membrane at room temperature for $30 \mathrm{~min}$. The specific proteins were visualized using Immobilon Western Chemiluminescent HRP Substrate (catalog no. WBKLS0500; EMD Millipore, Billerica, MA, USA) using a Bio-Rad Image station (Bio-Rad Laboratories, Inc., Hercules, CA, USA). Each experiment was repeated at least three times and representative results were presented.

Statistical analysis. Data are expressed as the mean \pm standard deviation and were analyzed with one-way analysis of variance followed by Dunnett's multiple comparison test. Statistical analysis was performed using SPSS software (version 19.0; IBM Corp., Armonk, NY, USA). $\mathrm{P}<0.05$ was considered to indicate a statistically significant difference.

\section{Results}

Sorafenib and luteolin combination induces synergistic cytotoxicity in human hepatocellular carcinoma cells. To investigate whether luteolin is able to enhance the anticancer activity of sorafenib, Hep3B cells were treated with increasing concentrations of luteolin $(5-20 \mu \mathrm{M})$ and a fixed concentration 
Table I. Synergistic interaction of sorafenib and luteolin in human hepatocellular carcinoma Hep3B cells.

\begin{tabular}{lcc}
\hline Sorafenib $(\mu \mathrm{mol} / \mathrm{l})$ & Luteolin $(\mu \mathrm{mol} / \mathrm{l})$ & CI \\
\hline 2 & 10 & 0.549 \\
3 & 10 & 0.674 \\
4 & 10 & 0.632 \\
2 & 5 & 0.841 \\
2 & 20 & 0.349 \\
\hline
\end{tabular}

The combination effect of sorafenib with luteolin was quantitatively evaluated using CI analysis. Briefly, the index is defined by the isobolar relation $\mathrm{CI}=\mathrm{a} / \mathrm{A}+\mathrm{b} / \mathrm{B}$, where $\mathrm{a}$ and $\mathrm{b}$ are the doses of chemicals 1 and 2 used in combination to achieve $\mathrm{x} \%$ drug effect, and $\mathrm{A}$ and $\mathrm{B}$ are the doses of the chemicals that applied singly to produce the same effect. $\mathrm{CI}<1,=1$, and $>1$ indicate synergism, additive effect, and antagonism, respectively. CI was calculated for combined treatment with indicated concentrations of sorafenib and luteolin. CI, combination index.

of sorafenib $(2 \mu \mathrm{M})$ for $72 \mathrm{~h}$. Cell death was measured using an LDH-release cytotoxicity assay. The results revealed that sorafenib at $2 \mu \mathrm{M}$ alone caused $\sim 10 \%$ cell death, and luteolin alone had little cytotoxicity with $<20 \%$ cell death even at the highest evaluated dose of $20 \mu \mathrm{M}$. However, combining luteolin and sorafenib increased cytotoxicity significantly in a dose-dependent manner with increasing concentrations of luteolin (Fig. 1A). Conversely, a similar potentiation of cytotoxicity was also identified with increasing concentrations of sorafenib $(2-4 \mu \mathrm{M})$ and a fixed luteolin dose $(10 \mu \mathrm{M})$ (Fig. 1B). The combinatory cytotoxic effect of luteolin and sorafenib was synergistic as evaluated using $\mathrm{CI}$ analysis as described previously (17) (Table I). In the human HCC cell line SMMC-7721, a similar dose-dependent synergism with fixed concentrations of sorafenib or luteolin was observed (Fig. 1C and D). These results suggest that the combination of luteolin and sorafenib was effective in sensitizing HCC cells to sorafenib-induced cytotoxicity.

Potentiated cytotoxity induces by the sorafenib and luteolin combination is achieved through apoptosis potentiation. Luteolin and sorafenib are able to induce apoptosis (18-21). Whether the enhanced cell death observed in HCC cells co-treated with luteolin and sorafenib was achieved through potentiation of apoptosis was then examined. Apoptosis in Hep3B cells treated with sorafenib in the absence or presence of luteolin was analyzed using flow cytometric assays. Early (B4) and late (B2) apoptotic cells were markedly increased in cells with sorafenib, and luteolin co-treatment compared with that in the cells treated with either agent alone (Fig. 2). Furthermore, the results demonstrated that activation of caspase 3 as demonstrated by the detection of the active form of caspase 3 was markedly potentiated in co-treated Hep3B cells (Fig. 3A). Consistently, the cleavage of the caspase-3 substrate PARP in the co-treated cells was also markedly enhanced (Fig. 3A). As expected, z-VAD-fmk significantly suppressed the enhanced cytotoxicity in Hep3B cells induced by co-treatment with sorafenib and luteolin (Fig. 3B). These
A

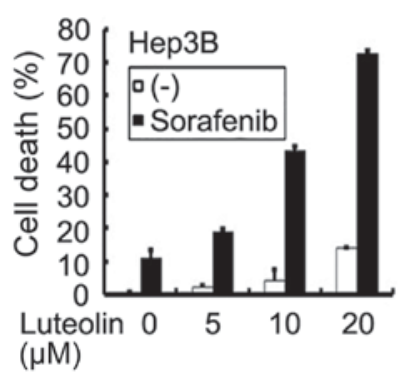

C

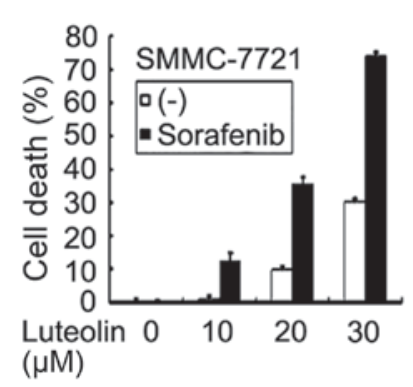

B
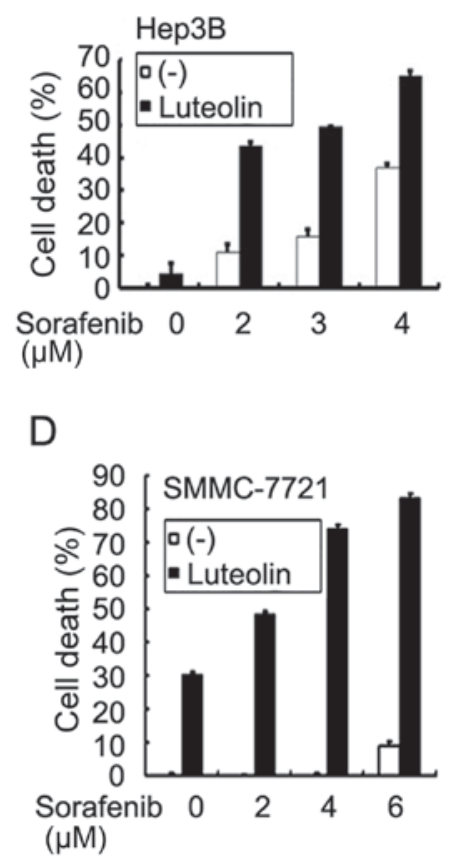

Figure 1. Combined treatment of sorafenib and luteolin induces synergistic cytotoxicity on hepatocellular carcinoma cells. (A) Hep3B cells were treated with increasing concentrations of luteolin $(5-20 \mu \mathrm{M})$ with or without a fixed concentration of sorafenib $(2 \mu \mathrm{M})$ for $72 \mathrm{~h}$. Cell death was measured by an assay based on the release of lactate dehydrogenase. (B) Hep3B cells were treated with increasing concentrations of sorafinib $(2-4 \mu \mathrm{M})$ with or without a fixed concentration of luteolin $(10 \mu \mathrm{M})$ for $72 \mathrm{~h}$. Cell death was measured as described in (A). (C) SMMC-7721 cells were treated with increasing concentrations of luteolin $(10-30 \mu \mathrm{M})$ with or without a fixed concentration of sorafenib $(4 \mu \mathrm{M})$ for $72 \mathrm{~h}$. Cell death was measured as described in (A). (D) SMMC-7721 cells were treated with increasing concentrations of sorafinib $(2-6 \mu \mathrm{M})$ with or without a fixed concentration of luteolin $(30 \mu \mathrm{M})$ for $72 \mathrm{~h}$. Cell death was measured as described in (A). The data is presented as the mean \pm standard deviation.

results suggest that the enhanced cytotoxicity induced by the sorafenib and luteolin combination was due to the potentiation of apoptosis.

Sorafenib and luteolin co-treatment potentiates the JNK activation pathway. The apoptotic cascade is tightly regulated in the cell by multistep regulation mechanisms, including multiple cellular signaling pathways. In order to dissect the mechanisms underlying enhanced cytotoxicity induced by luteolin and sorafenib combination, several inhibitors, including JNK inhibitor SP600125, ERK inhibitor U0126, P38 inhibitor SB203580, and ROS scavengers BHA and NAC were used to block corresponding pathways in sorafenib, and luteolin co-treated Hep3B cells. The results demonstrated that only SP600125 significantly inhibited the enhanced cytotoxicity induced by sorafenib and luteolin co-treatment, indicating that activation of JNK signaling pathway may be involved (Fig. 4A and B and data not shown). Indeed, while sorafenib- or luteolin alone slightly activated JNK as demonstrated, the sorafenib and luteolin co-treatment markedly potentiated JNK activation (Fig. 4C and data not shown). Altogether, these results suggest that the cytotoxic synergy of sorafenib and luteolin combination is induced by JNK-mediated apoptosis. 

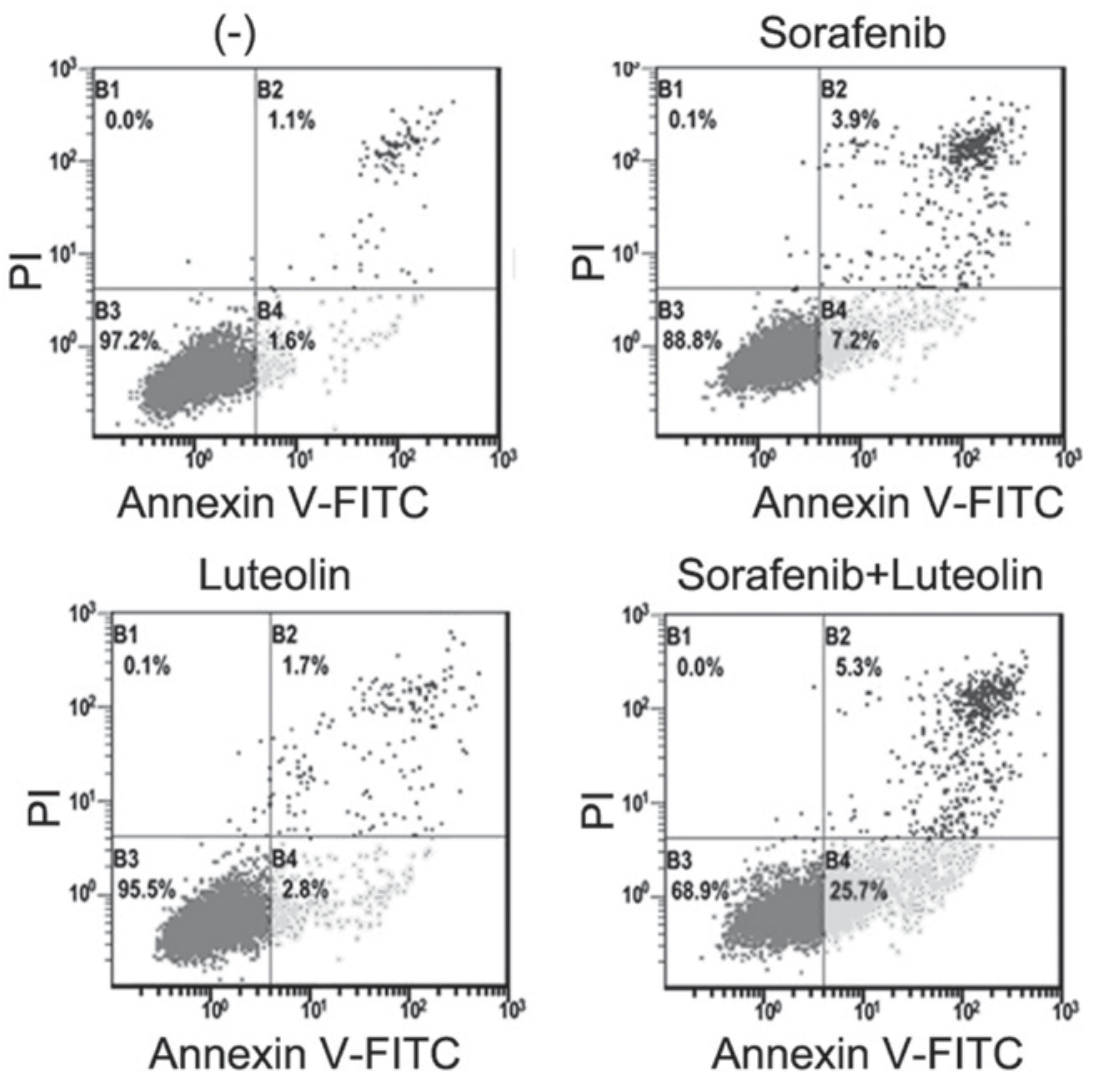

Figure 2. Apoptotic cells are significantly increased following sorafenib and luteolin co-treatment in hepatocellular carcinoma cells. Hep3B cells were treated with sorafenib $(3 \mu \mathrm{M})$ or luteolin $(10 \mu \mathrm{M})$ alone or in combination for $48 \mathrm{~h}$. The cells were then stained with Annexin V-FITC and PI followed by flow cytometry analysis. The percentage of cell population in B1, B2, B3, and B4 are presented, respectively. FITC, fluorescein isothiocyanate; PI, propidium iodide.

A

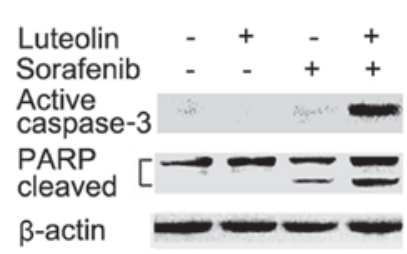

$\mathrm{B}$

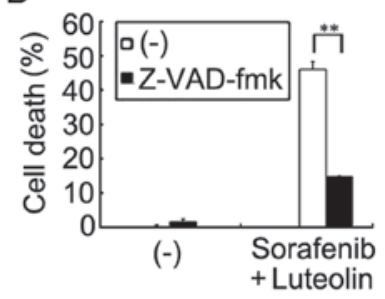

Figure 3. Activation of apoptotic pathway is potentiated in sorafenib and luteolin co-treated hepatocellular carcinoma cells. (A) Hep3B cells were treated with sorafenib $(3 \mu \mathrm{M})$ or luteolin $(10 \mu \mathrm{M})$ alone or in combination for $40 \mathrm{~h}$. Active caspase 3 and PARP were detected by western blotting. $\beta$-actin was detected as a loading control. (B) Hep3B cells were pretreated with Z-VAD-fmk $(20 \mu \mathrm{M})$ for 30 min or remained untreated, and then treated with sorafenib $(3 \mu \mathrm{M})$ and luteolin $(10 \mu \mathrm{M})$ for $72 \mathrm{~h}$. Cell death was measured as described in Fig. 1A. The data is presented as the mean \pm standard deviation; analysis of variance, $F=1306.473 ;{ }^{* *} \mathrm{P}<0.01$. PARP, poly (ADP-ribose) polymerase.

\section{Discussion}

In the present study, it was demonstrated that the combination of sorafenib and luteolin synergistically induced cytotoxicity in HCC cells. The enhanced cell death observed in sorafenib and luteolin co-treated cells was accompanied by potentiation of apoptosis. Furthermore, it was revealed that the sorafenib and luteolin combination markedly activated JNK, and the JNK inhibitor SP600125 significantly suppressed the synergistic cytotoxicity. Thus, potentiation of JNK-mediated

A

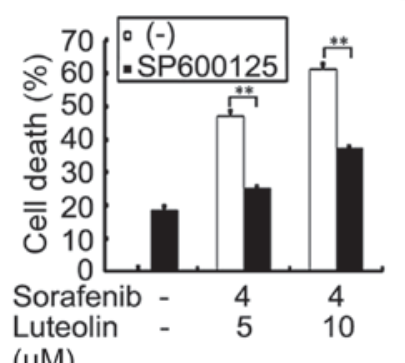

B

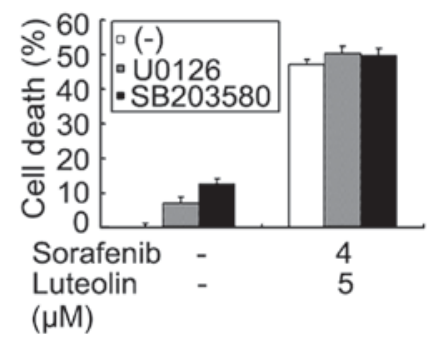

C

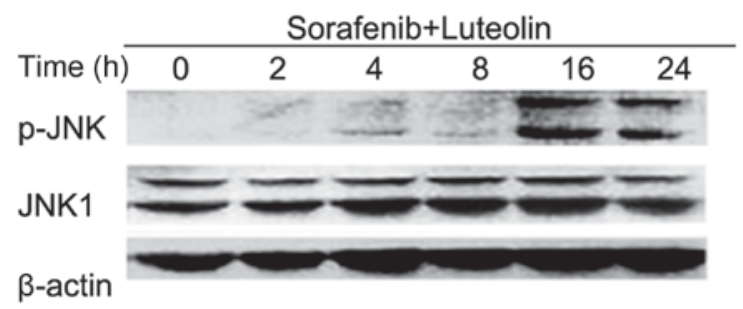

Figure 4. Sorafenib and luteolin co-treatment potentiates the JNK activation pathway. (A) Hep3B cells were pretreated with SP600125 $(10 \mu \mathrm{M})$ for $30 \mathrm{~min}$ or remained untreated, and then treated with sorafenib $(4 \mu \mathrm{M})$ and luteolin (5 $\mu \mathrm{M}$ or $10 \mu \mathrm{M})$ for $72 \mathrm{~h}$. (B) Hep3B cells were pretreated with U0126 $(10 \mu \mathrm{M})$ or SB $203580(10 \mu \mathrm{M})$ for $30 \mathrm{~min}$ or remained untreated, and then treated with sorafenib $(4 \mu \mathrm{M})$ and luteolin $(5 \mu \mathrm{M})$ for $72 \mathrm{~h}$. Cell death was measured as described in Fig. 1A. The data is presented as the mean \pm standard deviation; ${ }^{* *} \mathrm{P}<0.01$. (C) Hep3B cells were treated with sorafenib $(3 \mu \mathrm{M})$ and luteolin $(10 \mu \mathrm{M})$ for the indicated times. p-JNK and JNK1 were detected by western blotting. $\beta$-actin was detected as a loading control. P, phosphorylated; JNK, c-Jun N-terminal kinase. 
apoptosis contributed to the enhancement of cancer cell death caused by sorafenib and luteolin combination. To the best of our knowledge, this is the first report to demonstrate that the combination of sorafenib and luteolin is effective in killing human HCC cells.

As a candidate anticancer agent, luteolin has presented apoptotic effects in various tumor cells including lung cancer cells, hepatoma cells, pancreatic tumor cells, and leukemia cells with no significant toxic effects in normal cells $(12,22)$. The combination of luteolin with numerous conventional chemotherapeutics has been investigated in order to improve the anticancer efficacy of treatments $(15,19,20,23)$. Thus, it was considered that luteolin may be combined with targeted-therapy agent to provide efficacious treatment. To the best of our knowledge, the present study demonstrated for the first that the combination of luteolin and sorafenib kills human HCC cells in a synergistic manner. The results indicate that luteolin may be an ideal candidate for increasing the activity of sorafenib in HCC therapy, which warrants further in vivo investigation.

JNK is a main MAPK activated by extracellular stimuli and intracellular stresses. JNK can be induced by the MAP3K-MAP2K-JNK kinase signaling cascade or inactivated by a group of MAPK phosphatases, of which protein-tyrosine-phosphatase MKP1 (MKP1) is a major JNK suppressor $(24,25)$. Depending on the intensity and duration of the damage signal, JNK signaling may lead to either cell survival or cell death. JNK activation is often the target for cell death signaling induced by numerous chemotherapeutics, including lutoelin and sorafenib $(19,26,27)$. The results of the present study revealed that JNK activation was markedly potentiated in lutoelin and sorafenib co-treated cells, and that the JNK inhibitor (SP600125) was able to protect cells against cell death induced by the combination. Consistently, JNK was reported to be required for the synergistic cytotoxicity induced by the co-treatment of sorafenib and the proteasome inhibitor bortezomib (28). Our previous study demonstrated that luteolin triggers superoxide-dependent rapid degradation of the JNK suppressor MKP1 thus activating JNK (19). The mechanism underlying how the activation of JNK in lutoelin and sorafenib co-treated cells is promoted warrants further investigation.

\section{Acknowledgements}

The present study was supported by the National Natural Science Foundation of China (grant nos. 81172111 and 81372377) and the Science \& Technology Department of Sichuan Province, China (grant no. 2015JY0096).

\section{References}

1. Wilhelm S, Carter C, Lynch M, Lowinger T, Dumas J, Smith RA, Schwartz B, Simantov R and Kelley S: Discovery and development of sorafenib: A multikinase inhibitor for treating cancer. Nat Rev Drug Discov 5: 835-844, 2006.

2. Liu L, Cao Y, Chen C, Zhang X, McNabola A, Wilkie D, Wilhelm S, Lynch M and Carter C: Sorafenib blocks the $\mathrm{RAF} / \mathrm{MEK} / \mathrm{ERK}$ pathway, inhibits tumor angiogenesis, and induces tumor cell apoptosis in hepatocellular carcinoma model PLC/PRF/5. Cancer Res 66: 11851-11858, 2006.

3. Llovet JM, Ricci S, Mazzaferro V, Hilgard P, Gane E, Blanc JF, de Oliveira AC, Santoro A, Raoul JL, Forner A, et al: Sorafenib in advanced hepatocellular carcinoma. N Engl J Med 359: 378-390, 2008
4. Cheng AL, Kang YK, Chen Z, Tsao CJ, Qin S, Kim JS, Luo R, Feng J, Ye S, Yang TS, et al: Efficacy and safety of sorafenib in patients in the asia-pacific region with advanced hepatocellular carcinoma: A phase iii randomised, double-blind, placebo-controlled trial. Lancet Oncol 10: 25-34, 2009.

5. Kane RC, Farrell AT, Saber H, Tang S, Williams G, Jee JM, Liang C, Booth B, Chidambaram N, Morse D, et al: Sorafenib for the treatment of advanced renal cell carcinoma. Clin Cancer Res 12: 7271-7278, 2006.

6. Keating GM and Santoro A: Sorafenib: A review of its use in advanced hepatocellular carcinoma. Drugs 69: 223-240, 2009.

7. Gauthier A and Ho M: Role of sorafenib in the treatment of advanced hepatocellular carcinoma: An update. Hepatol Res 43: 147-154, 2013.

8. Birt DF, Hendrich S and Wang W: Dietary agents in cancer prevention: Flavonoids and isoflavonoids. Pharmacol Ther 90: 157-177, 2001.

9. Huang YT, Hwang JJ, Lee PP, Ke FC, Huang JH, Huang CJ, Kandaswami C, Middleton E Jr and Lee MT: Effects of luteolin and quercetin, inhibitors of tyrosine kinase, on cell growth and metastasis-associated properties in A431 cells overexpressing epidermal growth factor receptor. Br J Pharmacol 128: 999-1010, 1999.

10. Leung $\mathrm{HW}, \mathrm{Wu} \mathrm{CH}, \mathrm{Lin} \mathrm{CH}$ and Lee HZ: Luteolin induced DNA damage leading to human lung squamous carcinoma ch27 cell apoptosis. Eur J Pharmacol 508: 77-83, 2005.

11. Bagli E, Stefaniotou M, Morbidelli L, Ziche M, Psillas K, Murphy $\mathrm{C}$ and Fotsis T: Luteolin inhibits vascular endothelial growth factor-induced angiogenesis; inhibition of endothelial cell survival and proliferation by targeting phosphatidylinositol 3'-kinase activity. Cancer Res 64: 7936-7946, 2004.

12. Lin Y, Shi R, Wang X and Shen HM: Luteolin, a flavonoid with potential for cancer prevention and therapy. Curr Cancer Drug Targets 8: 634-646, 2008.

13. Yan J, Wang Q, Zheng X, Sun H, Zhou Y, Li D, Lin Y and Wang $X$ : Luteolin enhances tnf-related apoptosis-inducing ligand's anticancer activity in a lung cancer xenograft mouse model. Biochem Biophys Res Commun 417: 842-846, 2012.

14. Reipas KM, Law JH, Couto N, Islam S, Li Y, Li H, Cherkasov A, Jung K, Cheema AS, Jones SJ, et al: Luteolin is a novel p90 ribosomal S6 kinase (RSK) inhibitor that suppresses Notch4 signaling by blocking the activation of Y-box binding protein-1 (YB-1). Oncotarget 4: 329-345, 2013.

15. Yang MY, Wang CJ, Chen NF, Ho WH, Lu FJ and Tseng TH: Luteolin enhances paclitaxel-induced apoptosis in human breast cancer MDA-MB-231 cells by blocking STAT3. Chem Biol Interact 213: 60-68, 2014.

16. Wang X, Ju W, Renouard J, Aden J, Belinsky SA and Lin Y: 17-allylamino-17-demethoxygeldanamycin synergistically potentiates tumor necrosis factor-induced lung cancer cell death by blocking the nuclear factor-kappab pathway. Cancer Res 66 : 1089-1095, 2006.

17. Zhao L, Wientjes MG and Au JL: Evaluation of combination chemotherapy: Integration of nonlinear regression, curve shift, isobologram, and combination index analyses. Clin Cancer Res 10: 7994-8004, 2004.

18. Broecker-Preuss M, Müller S, Britten M, Worm K, Schmid KW, Mann K and Fuhrer D: Sorafenib inhibits intracellular signaling pathways and induces cell cycle arrest and cell death in thyroid carcinoma cells irrespective of histological origin or braf mutational status. BMC Cancer 15: 184, 2015.

19. Bai L, Xu X, Wang Q, Xu S, Ju W, Wang X, Chen W, He W, Tang H and Lin Y: A superoxide-mediated mitogen-activated protein kinase phosphatase-1 degradation and c-Jun NH(2)-terminal kinase activation pathway for luteolin-induced lung cancer cytotoxicity. Mol Pharmacol 81: 549-555, 2012.

20. Ju W, Wang X, Shi H, Chen W, Belinsky SA and Lin Y: A critical role of luteolin-induced reactive oxygen species in blockage of tumor necrosis factor-activated nuclear factor-kappaB pathway and sensitization of apoptosis in lung cancer cells. Mol Pharmacol 71: 1381-1388, 2007.

21. Kurosu T, Ohki M, Wu N, Kagechika H and Miura O: Sorafenib induces apoptosis specifically in cells expressing BCR/ABL by inhibiting its kinase activity to activate the intrinsic mitochondrial pathway. Cancer Res 69: 3927-3936, 2009.

22. Bai L, Chen W, Wang X, Ju W, Xu S and Lin Y: Attenuating smac mimetic compound 3-induced NF-kappaB activation by luteolin leads to synergistic cytotoxicity in cancer cells. J Cell Biochem 108: 1125-1131, 2009. 
23. Attoub S, Hassan AH, Vanhoecke B, Iratni R, Takahashi T, Gaben AM, Bracke M, Awad S, John A, Kamalboor HA, et al: Inhibition of cell survival, invasion, tumor growth and histone deacetylase activity by the dietary flavonoid luteolin in human epithelioid cancer cells. Eur J Pharmacol 651: 18-25, 2011.

24. Wagner EF and Nebreda AR: Signal integration by jnk and p38 mapk pathways in cancer development. Nat Rev Cancer 9: $537-549,2009$

25. Haagenson $\mathrm{K}$ and Wu GS: Mitogen activated protein kinase phosphatases and cancer. Cancer Biol Ther 9: 337-340, 2010.

26. Choi AY, Choi JH, Yoon H, Hwang KY, Noh MH, Choe W, Yoon KS, Ha J, Yeo EJ and Kang I: Luteolin induces apoptosis through endoplasmic reticulum stress and mitochondrial dysfunction in Neuro-2a mouse neuroblastoma cells. Eur J Pharmacol 668: 115-126, 2011.
27. Ou DL, Shen YC, Yu SL, Chen KF, Yeh PY, Fan HH, Feng WC, Wang CT, Lin LI, Hsu C and Cheng AL: Induction of DNA damage-inducible gene GADD45beta contributes to sorafenib-induced apoptosis in hepatocellular carcinoma cells. Cancer Res 70: 9309-9318, 2010.

28. Yu C, Friday BB, Lai JP, Yang L, Sarkaria J, Kay NE, Carter CA, Roberts LR, Kaufmann SH and Adjei AA: Cytotoxic synergy between the multikinase inhibitor sorafenib and the proteasome inhibitor bortezomib in vitro: Induction of apoptosis through Akt and c-Jun NH2-terminal kinase pathways. Mol Cancer Ther 5: 2378-2387, 2006 